\title{
The In vivo Effects of Caffeine on the Hypoglycemic Activity of Gliclazide and Metformin in Healthy Rats
}

\author{
Mohammad Mohiuddin, Md. Shah Amran and Md. Amjad Hossain \\ Department of Pharmaceutical Chemistry, Faculty of Pharmacy, \\ University of Dhaka, Dhaka-1000, Bangladesh
}

\begin{abstract}
An in vivo study has been conducted to observe the effects of caffeine on the hypoglycemic activity of gliclazide and metformin HCI in rats. For this, healthy rats weighing about $250 \pm 25 \mathrm{~g}$ were used and the blood sugar levels were measured after administration of a drug alone and in combination. Diabetes was induced in rats by administration of alloxan at a dose of $40 \mathrm{mg} / \mathrm{kg}$ body weight. Drugs were administered in rats and were observed for four weeks. The blood sugar levels were estimated in two stages; firstly, after two week and secondly, after four week of administration of drug. It was found that the hypoglycemic activities of both gliclazide and metformin $\mathrm{HCl}$ were potentiated by concurrent application of caffeine and either of the drugs, but the extent of potentiation was more in case of metformin HCI as evident from the blood sugar level of rats.
\end{abstract}

Key words: Caffeine, Gliclazide, Metformin, Diabetes, Alloxan, Blood sugar, Rats.

\section{INTRODUCTION}

Caffeine is a methylxanthine that inhibits the enzyme phosphodiesterase and has an antagonistic effect on central adenosine receptors. It is a stimulant of the CNS, particularly the higher centre, and it can produce a condition of wakefulness and increased mental activity. It may also stimulate the respiratory centre, increasing the rate and depth of respiration. Caffeine facilitates the performance of muscular work and increases the total work, which can be performed by a muscle. Caffeine is used as a mild CNS stimulant in usual doses of 50 to $100 \mathrm{mg}$ by mouth, although doses of up to $200 \mathrm{mg}$ may be used. It is also frequently included in oral analgesic preparations with aspirin, paracetamol, or codeine in unit doses of 15 to $65 \mathrm{mg}$ although its clinical benefit

Correspondence to:

Md. Amjad Hossain

E-mail: arshi15edu@bangla.net is debated. It is sometimes given with ergotamine in preparations for the treatment of migraine; usually in unit doses of $100 \mathrm{mg}$. Caffeine is absorbed readily after oral administration and is widely distributed throughout the body. It is also absorbed through the skin. Caffeine is metabolized almost completely in the liver via oxidation, demethylation, and acetylation, and is excreted in the urine and other metabolites with only about $1 \%$ unchanged. ${ }^{1,2}$ Gliclazide is a sulfonylurea antidiabetic agent. It is given by mouth in the treatment of type-II diabetes mellitus and has a duration of action of 12 to 14 hours. Gliclazide is readily absorbed from the gastrointestinal tract. It is extensively bound to plasma proteins. The half-life is about 10 to 12 hours. It is extensively metabolized in the liver to metabolites that have no significant hypoglycemic activity. The metabolites and a small amount of unchanged drug are excreted in the urine. ${ }^{3}$ 
Metformin Hydrochloride is a biguanide antidiabetic agent. It is given by mouth in the treatment of type II diabetes mellitus, and is the drug of first choice in obese patients. Metformin Hydrochloride is slowly and incompletely absorbed from the gastrointestinal tract. Once absorbed plasma protein binding is negligible, and it is excreted unchanged in the urine. The plasma elimination halflife is reported to range from about 2 to 6 hours after oral administration. Metformin is distributed into breast milk in small amounts. ${ }^{4,5}$

The studies relating to influence of a well known and easily available drug like caffeine on the properties of antidiabetic molecules may become fruitful in various ways. The area of studies on drug interaction correlates all the disciplines of drug management and health care systems relevant to the contemporary practice of medicine. Knowledge of drug interactions may allow early recognition and prevention of adverse consequences. Adverse drug interactions can cause a loss in therapeutic activity that may lead to toxicity or unexpected increase in pharmacological activity of a drug due to alteration of absorption or other biochemical processes. Further, the study of a drug interaction is an important part in the field of drug research, especially drug design and development of newer drugs. ${ }^{6}$

The antidiabetic drugs are enlisted as essential drugs in our country and caffeine is also an important drug and therefore this work may help us to collect information about improvement of their effect. Therefore the aim of the present study was to observe the effect of Caffeine on the hypoglycemic activity of Gliclazide and Metformin HCI and thus to infer about the possibility of combination therapy.

\section{MATERIALS AND METHODS}

Materials. Caffeine, Gliclazide and Metformin HCI were kind gifts from the Orion Laboratories Ltd., Dhaka, Bangladesh.

Reagents. Disodium oxalate (reagent grade), $10 \%$ Na-tungstate, arsenic molybdate (reagent grade),
Fehling Solution, Sulphuric acid (reagent grade), 1\% glucose solution, alloxan, demineralized water.

Equipments. UV-visible Spectrometer (Model No. UV-1601, Shimadzu, Japan), pH Meter (Mettler Toledo, Switzerland), Power Sonic (Model No. 510, Seoul, Korea), Dubnoff metabolic shaking incubator (GCA corporation, USA), centrifuge machine, colorimeter and microsyringe.

Animals. Thirty healthy rats weighing about $250 \pm 25 \mathrm{~g}$ were used as the experimental animals for the in vivo experiment. The animals were collected from the International Centre for Diarrhoeal Disease Research, Bangladesh (ICDDR, B).

Preparation of standard solutions. Caffeine, gliclazide and metformin HCI were dissolved in demineralized water separately. These stock solutions were diluted to desired strengths by buffer solution (pH 7.4) to get the working standard solution for each drug.

Preparation of standard curve. For preparing a standard curve, 20, 40, 60, 80, 100 and $120 \mu \mathrm{g} / \mathrm{ml}$ glucose solutions were prepared by using supplied $1 \%$ glucose solution. $1 \mathrm{ml}$ of each solution was taken in different marked tubes by $1 \mathrm{ml}$ pipette containing 1 $\mathrm{ml}$ of Fehling solution (Fehling solution was prepared in sito by mixing $25 \mathrm{ml}$ of solution A and $1 \mathrm{ml}$ of solution of $\mathrm{B}$ ) and then heated in a water bath for 30 minutes. Then the solutions were cooled in a beaker of cold water and one mililiter of arsenic molybdate and $22 \mathrm{ml}$ of demineralized water were added and mixed well. Then the absorbance was measured at $520 \mathrm{~nm}$ and a standard curve was prepared by plotting absorbance versus concentration of glucose (Figure 1).

Test animals and administration of drugs. Thirty adult rats aged 2-3 months, each weighing $250 \pm 25 \mathrm{~g}$ of both sexes were used in the in vivo experiment. ${ }^{7-9}$ They were divided into 6 groups each having 5 rats (marked as I, II, III, IV, V and VI). Group I was the control group. Drugs were administered in rats and were observed for four weeks. Diabetes was induced in rats by administration of alloxan. 
Group I. Each rat received $2 \mathrm{ml}$ of intraperitoneal injection of normal saline for three consecutive days.

Group II. Each rat received $2 \mathrm{ml}$ of intraperitoneal injection of alloxan $^{13}$ as a dose of 40 $\mathrm{mg} / \mathrm{kg}$ body weights for three consecutive days.

Group III. They received alloxan injection as in group II. On the fourth day (after induction of diabetes as indicated by hyperglycemia) each rat received gliclazide solution as a dose of $1.60 \mathrm{mg} / \mathrm{kg}$ body weights.

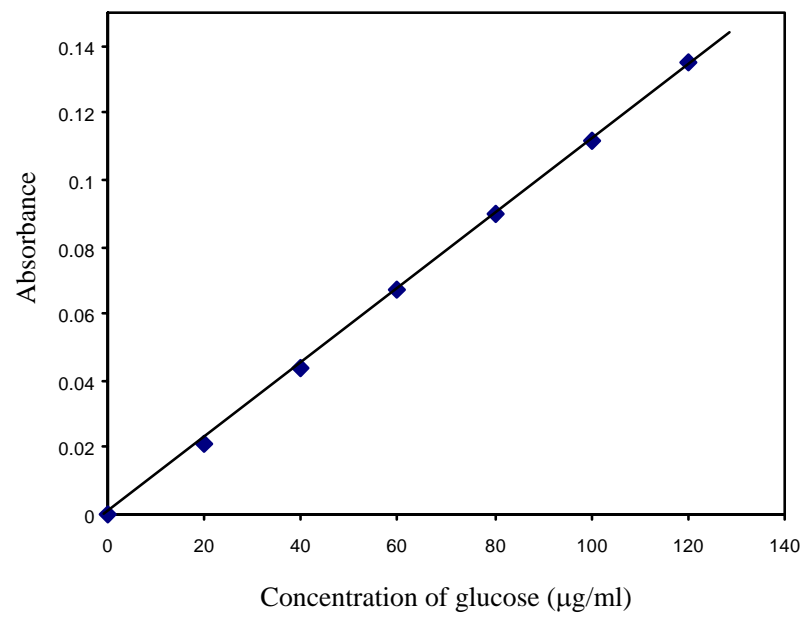

Figure 1. Standard curve for the estimation of blood sugar level

Group IV. They received alloxan injection as in group II. On the fourth day (after induction of diabetes as indicated by hyperglycemia) each rat received a mixture of gliclazide and caffeine solution as a dose of $1.60 \mathrm{mg} / \mathrm{kg}$ and $1.30 \mathrm{mg} / \mathrm{kg}$ body weights, respectively.

Group V. They also received alloxan injection. On the fourth day each rat received Metformin HCI solution as a dose of $10 \mathrm{mg} / \mathrm{kg}$ body weights.

Group VI. As above, they also received alloxan injection and mixture of metformin HCI and caffeine solution as a dose of $10 \mathrm{mg} / \mathrm{kg}$ and $1.30 \mathrm{mg} / \mathrm{kg}$ body weights, respectively.

Estimation of blood sugar level in rat. In all experimental cases, blood was collected after overnight fasting for estimation of sugar. Blood samples $(1 \mathrm{ml})$ were collected in centrifuge tubes by disposable plastic syringe from the vein in the tail and centrifuged to collect the serum.

1. Required test tubes were taken and marked in numbers for each group.

2. Serum was poured into a small glass tube containing disodium oxalate and shaken well to inhibit the coagulation.

3. In each tube $0.5 \mathrm{ml}$ of serum and $8.5 \mathrm{ml}$ of demineralized water were taken. With these 0.5 $\mathrm{ml}$ of $10 \% \mathrm{Na}$-tungstate and $0.5 \mathrm{ml} 2 / 3 \mathrm{~N}$ $\mathrm{H}_{2} \mathrm{SO}_{4}$ were mixed properly for complete precipitation of protein.

4. To sediment the precipitated proteins and to obtain supernatant fluid, the mixture was centrifuged at a maximum speed for 5 minutes.

5. $1 \mathrm{ml}$ of supernatant fluid was taken by $1 \mathrm{ml}$ pipette into the test tubes containing $1 \mathrm{ml}$ of Fehling solution (Fehling solution was prepared in sito by mixing $25 \mathrm{ml}$ solution $\mathrm{A}$ and $1 \mathrm{ml}$ of solution of B) and then heated in a water bath for 30 minutes. The solutions were cooled in a beaker of cold water.

6. $1 \mathrm{ml}$ of arsenic molybdate and $22 \mathrm{ml}$ of demineralized water were added and mixed well.

7. The absorbance was measured at $520 \mathrm{~nm}$ and the concentration of glucose was determined by using the standard curve.

Statistical analysis. The results were expressed as mean \pm s. e. $\mathrm{m}$. values for each experiment. Differences in mean values between experimental groups were analyzed by unpaired ' $\mathrm{t}$ ' test. A probability value less than $0.05(\mathrm{p}<0.05)$ was defined to be significant.

\section{RESULTS AND DISCUSSION}

Blood sugar levels of rats were measured after administration of a drug (gliclazide or metformin $\mathrm{HCl}$ ) alone and in combination with caffeine. The blood sugar levels were estimated in two stages: after two weeks and after four weeks of the administration of drug. The treated rats showed blood sugar levels of 
$253 \pm 3.1 \mathrm{mg} / 100 \mathrm{ml}$ and $304 \pm 2.7 \mathrm{mg} / 100 \mathrm{ml}$ respectively after two weeks and four weeks of alloxan application. These results were in consistent with other workers. ${ }^{10}$

Normal blood sugar level was $72 \pm 2.9$ $\mathrm{mg} / 100 \mathrm{ml}$. After induction of diabetes by administration of alloxan, the blood sugar level raised to $253 \pm 3.1 \mathrm{mg} / 100 \mathrm{ml}$. At this stage, when gliclazide alone was administered, the blood sugar level decreased to $136 \pm 5.1 \mathrm{mg} / 100 \mathrm{ml}$ and when gliclazide was administered with caffeine the blood sugar level decreased to $122 \pm 4.5 \mathrm{mg} / 100 \mathrm{ml}$. On the other hand, when metformin $\mathrm{HCl}$ alone was administered, the blood sugar level decreased to 128 $\pm 5.6 \mathrm{mg} / 100 \mathrm{ml}$ and when metformin $\mathrm{HCl}$ was administered with caffeine the blood sugar level decreased to $116 \pm 5.0 \mathrm{mg} / 100 \mathrm{ml}$ (Figure 2) after two weeks of treatment.

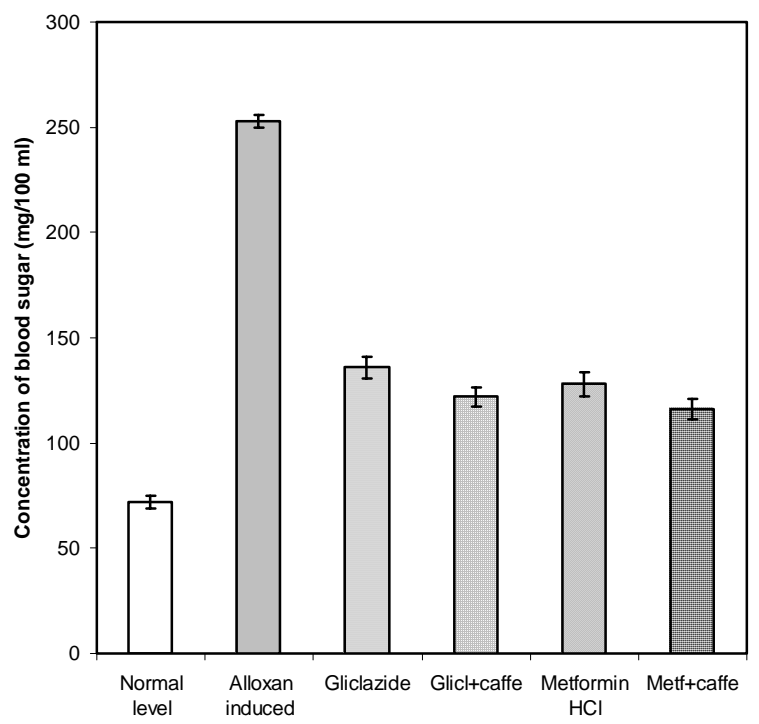

Figure 2. Comparison of blood sugar level observed in rats after two weeks of the administration of drugs. The results are shown as mean \pm s.e.m. $(n=5)$.

Again in the other experiment, the normal blood sugar level was $74.2 \pm 2.6 \mathrm{mg} / 100 \mathrm{ml}$. After induction of diabetes by administration of alloxan, the blood sugar level raised to $304 \pm 2.7 \mathrm{mg} / 100 \mathrm{ml}$. At this stage, when gliclazide alone was administered, the blood sugar level decreased to 130 $\pm 6.4 \mathrm{mg} / 100 \mathrm{ml}$ and when gliclazide was administered with caffeine the blood sugar level decreased to $114 \pm 3.4 \mathrm{mg} / 100 \mathrm{ml}$. On the other hand, when metformin $\mathrm{HCl}$ alone was administered, the blood sugar level decreased to $122 \pm 5.1 \mathrm{mg} / 100 \mathrm{ml}$ and upon administering metformin $\mathrm{HCl}$ with caffeine the blood sugar level decreased to $98 \pm 5.4 \mathrm{mg} / 100$ $\mathrm{ml}$ (Figure 3) after four weeks of treatment.

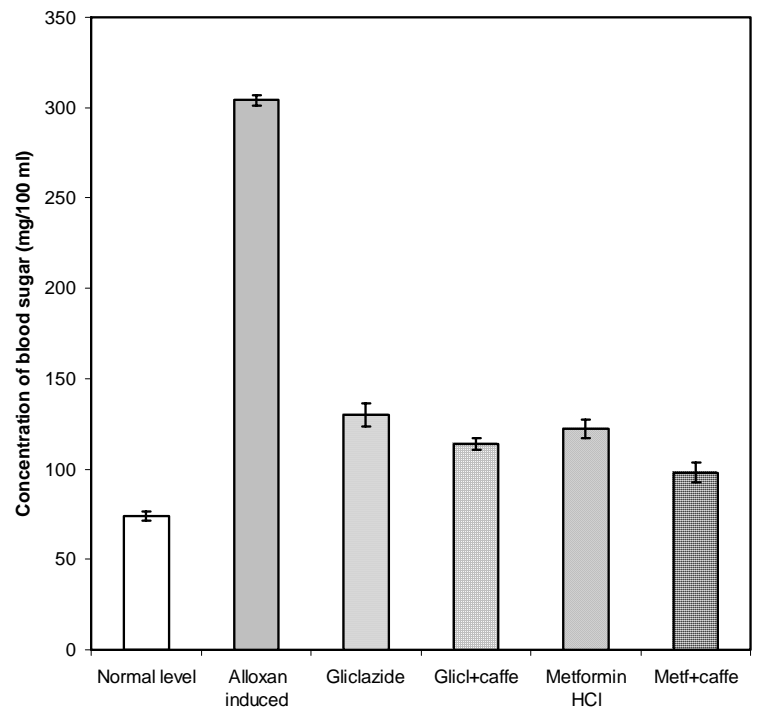

Figure 3. Comparison of blood sugar level observed in rats after four weeks of the administration of drugs. The results are shown as mean \pm s.e.m. $(n=5)$.

Amran et al. ${ }^{11}$ studied the in vitro effects of glipizide and gliclazide on the protein binding and plasma concentration of ibuprofen. It was found that ibuprofen decreased the protein binding of glipizide and gliclazide which may be due to the displacement of glipizide and gliclazide by the ibuprofen from the protein binding sites.

So, it can be concluded that the hypoglycemic activity of both gliclazide and metformin $\mathrm{HCl}$ is potentiated by concurrent application of caffeine. In our study we found that caffeine did not have any hypglycemic property. However, it was observed that the influence of caffeine on the hypoglycemic activity of metformin $\mathrm{HCl}$ was stronger than that on gliclazide. It may be mentioned that caffeine does not possess any hypoglycemic property. The potentiation of the antidiabetic properties of gliclazide and metformin $\mathrm{HCl}$ may be due to relaxation effect of caffeine on smooth muscles of the rat as well as some 
sort of modification of the molecular conformations of the antidiabetic agents. We have also obtained the evidence of interaction (complexation) of caffeine with these two drugs in a separate study ${ }^{12}$, where it has been revealed that caffeine formed 1:1 complexes with gliclazide and metformin $\mathrm{HCl}$.

\section{CONCLUSION}

Multiple drug therapy is a useful and common practice in modern medical science, where two or more drugs are administered concurrently. The results in this study have shown that caffeine can enhance hypoglycemic effect of both gliclazide and metformin $\mathrm{HCl}$ in rats. The data obtained would help us to suggest that gliclazide as well as metformin $\mathrm{HCl}$ may result into compatible combination therapies with caffeine. However trials in higher animals and human are necessary.

\section{ACKNOWLEDGEMENT}

The authors thank the 'International Centre for Diarrhoeal Disease Research, Bangladesh (ICDDR, B) for supplying the test animals and 'Orion Laboratories Ltd.’, Dhaka, Bangladesh, for supplying the test drugs.

\section{REFERENCES}

1. Sawynok, J. 1995. Pharmacological rationales for the clinical use of caffeine. 49, 37-50.

2. Martindale: 2005. The Complete Drug Reference: $34^{\text {th }}$ edition; Pharmaceutical press: p-332, 342, 783.

3. Kobayashi, K. et al. 1984. Pharmacokinetics of gliclazide in healthy and diabetic subjects. J. Pharm. Sci. 73, 1684-1687.

4. Scheen, A. J. 1996. Clinical pharmacokinetics of metformin. Clin Pharmacokinet. 30, 359-371.
5. Sambol, N.C. et al. 1996.Pharmacokinetics and Pharmacodynamics of metformin in healthy subjects and patients with non-insulin-dependent-diabetes- mellitus. $J$. Clin. Pharmacol. 36, 1012-1021.

6.. Philip, D.H. and Horn, J.R. 1989. Drug interactions. In: Clinical significance of drug- drug interaction, $6^{\text {th }}$ edition, Philadelphia, pp. 5-13.

7. Zaman, M.R., Amran, M.S. and Hossain, M.A. 1999. In vivo study of effects of nifedipine, Ketotifen fumerate and potassium nitrate on plasma concentration of dilitazem in rabbits. Pakistan J. Pharmacol. 16, 57-61.

8. Akhter, B., Amran, M.S. and Hossain, M.A. 2001. In vivo evalution of famotidine and prochlorperazine maleate on the serum concentration of nifedipine in rabbits. Pakistan $J$. Pharmacol. 18, 23-29.

9. Amran, M.S., Hashimoto, K. and Homma, N. 2004. Effects of sodium-calcium exchange inhibitors, KB-R7943 and SEA0400, on aconotine-induced arrhythmias in guinea pigs in vivo, in vitro and in computer simulation studies. $J$. Pharmacol. Exp. Ther. 310, 83-89.

10. Kobayashi K, Kimura M, Sakoguchi T, Hase A, Matsuoka A, Kaneko S: 1984. Pharmacokinetics of Gliclazide in healthy and diabetic subjects. J. Pharm. Sci. 73, 1684-1687.

11. Amran, M.S., Baki, M.A. and Hossain, M.A. 2009. In vitro and in vivo effects of glipizide and gliclazide on the protein binding and plasma concentration of ibuprofen. Presented at the 31st Annual Conference of Bangladesh Chemical Society, University of Dhaka, Dhaka, Bangladesh. 30 Jan.01 Feb. 2009.

12. Mohiuddin, M. and Hossain, M.A. 2008. A study on the interaction of gliclazide and metformin $\mathrm{HCl}$ with caffeine in the aqueous media. M. Pharm. Thesis, Department of Pharmaceutical Chemistry, Faculty of Pharmacy, University of Dhaka, Dhaka-1000, Bangladesh.

13. Solomon J., Bulkley R.J., Mayer J., 1974. Effect of alloxan on blood glucose, islet beta cell granulation, body weight and insulin resistance of ob/ob mice. Diabetologia 10, 709-714. 